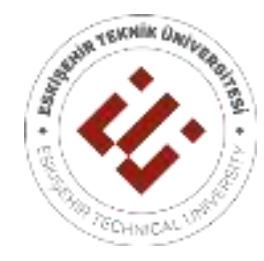

ESKISSEHİR TECHNICAL UNIVERSITY JOURNAL OF SCIENCE AND TECHNOLOGY A- APPLIED SCIENCES AND ENGINEERING

8th International Fiber and Polymer Research Symposium

(8th ULPAS) - Special Issue 2021

2021, Vol.22 pp. 28-38, DOI:10.18038/estubtda.978207

\title{
STRETCHABLE PIEZORESISTIVE SENSORS WITH GRAPHENE AND POLYANILINE COATED WOVEN POLYESTER FABRICS
}

\author{
Meryem ÇETINOĞLU 1 (i), Gizem FINDIK ${ }^{1}$ (i), \\ Ömer Faruk ÜNSAL 1 (i), Ayşe ÇELİK BEDELOĞLU ${ }^{1}$ * (iti
}

${ }^{1}$ Polymer Materials Engineering Department, Faculty of Engineering and Natural Sciences, Bursa Technical University, Bursa, Turkey

\begin{abstract}
Wearable electronics, which include wearable sensors, energy generating textiles, textile based energy storage devices, etc. is a rising trend on materials science and textile researches for last a few decades. Textile based flexible capacitors, nanogenerators, motion sensors, photovoltaic cells have been studied greatly and these devices getting commercial for last a few years. Strain is the concept that expresses mechanical deformation rate for a material. Strain sensors are smart materials, which works according to piezoelectric and piezoresistive mechanism to determine the mechanical deformation rate of materials, that can be used in mechanical characterization, structural quality control and, more recently, wearable electronics. Piezoresistive effect defines the electrical conductivity (or resistivity) changes of a material under mechanical stress. Especially on nanocomposite based strain sensors, contact between conductive nanoparticles is interrupted and this dissociation causes the resistivity change under mechanical effect. We have used a commercial, highly flexible woven fabric as base material and its flexibility provided the stretchable character for developed sensor fabric. Combining its flexibility with conductivity of graphene and/or polyaniline, promising resistivity values were observed. Furthermore, flexible nature of graphene and brittle behavior of polyaniline clearly seen in electro-mechanical characterization. The developed fabric strain sensor can be used in smart textiles and future applications for wearable electronics.
\end{abstract}

Keywords: Strain sensor, Graphene, Conductive polymer, Smart textiles, Electronic fabrics

\section{INTRODUCTION}

With the improvement of wearable technology flexible smart wearable devices have been significant in the daily life. One of these devices, flexible strain sensors have been attracted a lot of interest due to their highly flexibility on surface coating and with the purpose of observing structural stability of wearable electronics, smart devices, smart fabrics, soft robotic and materials lately [1]. Fabrics are useful for different implementations by the developing technology and taking the human body shape for smart textile applications [2-4]. In this qualifications some of the applications are based on elastic deformation ability. Strain sensors are the devices which have signal generation ability under mechanical forces [5].

Generally, various fibers, yarns and fabrics can be used to develop wearable devices. Lately, researches on flexible sensors based on successfully-produced fabrics for wearable devices are becoming more important [6]. For that reason, flexible fabrics with lycra is eligible for obtaining wearable flexible sensors that have high performance and easy production processes. Graphene and its derivatives are commonly used for flexible sensors in literature [7,8], due to their remarkable electrical and mechanical properties [9]. Graphene is a material one-carbon-atom-thick, structure of hexagonally arranged, sp2hybridized carbon atoms. In addition to its remarkable 2-dimensional structure, graphene also stands out with its low electrical resistance and thermal conductivity $(31 \Omega / \mathrm{m} 2$ resistivity, $5300 \mathrm{~W} / \mathrm{m} . \mathrm{K})$, and high mechanical properties compared to its thickness at the atomic level[10-12]. On the other hand, polyaniline is a conducting polymer that has a wide range of usage area, affordable, easily-producible and multi conductive $[13,14]$. It can be synthesized with oxidative chemical or electrochemical

*Corresponding Author: ayse.bedeloglu@btu.edu.tr

Received: 03.08.2021 Published: 30.11.2021 
oxidations by polymerization of aniline monomer, in acidic aqueous medium [15, 16]. PANI coating of textile materials is a low cost and efficient method by oxidative polymerization and PANI coated textiles have many usage areas such as; sensors electromagnetic protection, static charge distribution [17]. PANI can be implemented in coating the surface of polyester, nylon, wool, acrylic, cotton, silica and glass. It is a widely used polymer among conductive materials in addition to its unique properties such as high thermal, electrical and environmental/chemical stability as well as its reasonable cost. PANI is widely used with different materials such as graphene and its derivatives to gain synergistic mechanical or electrical effect due to its poor performance in energy storage, contra ion addition/discharge and charge/discharge cycle life and weak mechanical properties [13, 14, 18].

Thanks to easy applicable to textiles, low cost, unique electrical and mechanical properties, and synergistic effect of PANI and graphene (or derivatives), there too many researches on PANI/graphene based electronic textiles. Fabric electrodes, textile based supercapacitors, solar cells, sensors, and energy generators [19-23].

In this study, conductive fabrics were obtained by dip-coating and in-situ oxidative polymerization processes on elastic polyester fabric. Reduced graphene oxide (rGO), PANI or both are successfully coated onto the textile surface. The electrical resistances of the obtained conductive fabrics were measured simultaneously under tensile stress, and the feasibility of different coatings in sensor applications was evaluated by revealing the change in their electrical resistance with elongation. The fabrics also characterized by differential scanning calorie spectrometry (DSC), Fourier-transform infrared spectrophotometry (FT-IR), UV-VIS spectrophotometry and scanning electron microscopy (SEM).

\section{MATERIALS AND METHODS}

\subsection{Materials}

For graphene oxide (GO) production, graphite (Merck), phosphoric acid (85\% Sigma-Aldrich), sulfuric acid (98\% Merck), hydrogen peroxide (35\%, Sigma-Aldrich), hydrochloric acid (37\%, Fischer Chemicals), ethyl alcohol (ISOLAB); for reduction process, hydrazine hydrate (55\%, Sigma) and for polyaniline synthesis aniline (99,9\%, Sigma-Aldrich), hydrochloric acid (37\%, WVR Chemicals) and ammonium persulfate (APS) (98\%, Sigma) were purchased.

\subsection{Methods}

Polyester fabric was washed at $30^{\circ} \mathrm{C}$ in a home-type washing machine then dried. In order to purify the dried fabric from the ions in the detergent or mains water, it was kept in distilled water for 30 minutes and dried again. Then it was cut into $6 \mathrm{~cm} \times 20 \mathrm{~cm}$ dimensions.

\subsection{1. rGO coating of fabrics}

In this study, graphene oxide is used as graphene precursor material thanks to its highly homogenous dispersion ability in water. Graphene oxide (GO) was produced by wet chemical process, according to Improved Hummers' Method [24]. GO particles are purified by distilled water and centrifuged repeatedly in order to removal of residual ions and acid molecules which come from synthesis step. Purified GO was dried in fume hood in room conditions. GO dispersion was prepared at a concentration of $1 \mathrm{mg} / \mathrm{mL}$ by homogenizing solid GO in distilled water with an ultrasonic homogenizer. $6 \mathrm{~cm} \times 20$ $\mathrm{cm}$ fabrics are immersed in $\mathrm{GO} /$ water dispersion and kept in dispersion for 10 minutes for each coating and then dried at $50^{\circ} \mathrm{C}$ for 30 minutes. Fabrics were stored by coating once, twice and three times. Once, 
twice or three times $\mathrm{GO}$ coated fabrics were reduced in $0.3 \mathrm{M}$ hydrazine hydrate solution at $95^{\circ} \mathrm{C}$ for 3 hours [25].

\subsubsection{PANI coating of fabrics}

rGO coated or neat polyester fabrics were coated with polyaniline by in situ polymerization method. PANI synthesis was carried out in accordance with the method in the literature with presence of rGO coated or neat polyester fabric [26]. Firstly, aniline was dissolved in $1 \mathrm{M} \mathrm{HCl}$ solution. The reaction flask was cooled between $0-5{ }^{\circ} \mathrm{C}$ and the fabrics were immersed in the reaction flask by wrapping it around a cylinder like shaped apparatus to keep it stable and coat homogeneously. Ammonium persulfate used as initiator was also dissolved in $1 \mathrm{M} \mathrm{HCl}$ and added dropwise to the reaction flask. APS:aniline molar ratio was determined as 1:4. The reaction was continued for 3 hours from the start of addition of the initiator. Since the rGO coated fabrics shrink due to the heat during GO reduction process, the neat fabric was also boiled at the same temperature. All of samples were named in Table 1.

Table 1. Sample names of fabrics with different coatings

\begin{tabular}{cc}
\hline Sample Name & Coatings \\
\hline rGP1 & Once rGO + PANI \\
rGP2 & Twice rGO + PANI \\
rGP3 & 3 times rGO + PANI \\
P & PANI \\
rG2 & Twice rGO \\
rG3 & 3 times rGO \\
GO1 & Once GO \\
GO2 & Twice GO \\
GO3 & 3 times GO \\
\hline
\end{tabular}

\subsubsection{Characterization}

In order for determining the elastic region of the fabric, a tensile test was applied to fabric (SHIMADZU - AGS-X). It was then subjected to tensile test to examine its electro-mechanical sensing features. The fabric sensor was held for 5 seconds at every $5 \%$ elongation. Electrodes were mounted onto fabrics and connected a multimeter (KEITHLEY 2400) and resistance values were recorded at this time. The test speed was determined as $5 \mathrm{~mm} / \mathrm{s}$. The effect of the coating was observed by subjecting the raw fabric and rGO and/or PANI coated fabrics to FT-IR spectrophotometry (Nicolet- IS50), UV-VIS spectrophotometry, DSC methods. Fiber morphology and coated areas of fabrics also examined by SEM (Carl Zeiss / Gemini 300) device with different magnifications.

\section{RESULTS}

FT-IR analysis were performed between $400 \mathrm{~cm}-1$ and $4000 \mathrm{~cm}-1$ with Attenuated Total Reflectance (ATR) unit. As the result of FT-IR analyzes it was observed that the characteristic polyester peaks between $1750 \mathrm{~cm}-1$ and $400 \mathrm{~cm}-1$ were visible after coating, although their intensity decreased (Figure 1). It was determined that the $\mathrm{C}-\mathrm{H}$ peaks at approximately $2900 \mathrm{~cm}-1$ disappeared with the coating process. The peaks at $1711 \mathrm{~cm}-1$ represents from stretch of carbonyl groups, at $1409 \mathrm{~cm}-1$ comes from aromatic groups on backbone, $1235 \mathrm{~cm}-1$ is $\mathrm{C}-\mathrm{O}$ stretching, and $720 \mathrm{~cm}-1$ is $\mathrm{C}-\mathrm{H}$ bending vibrations, respectively (Figure 1) [27-29]. 


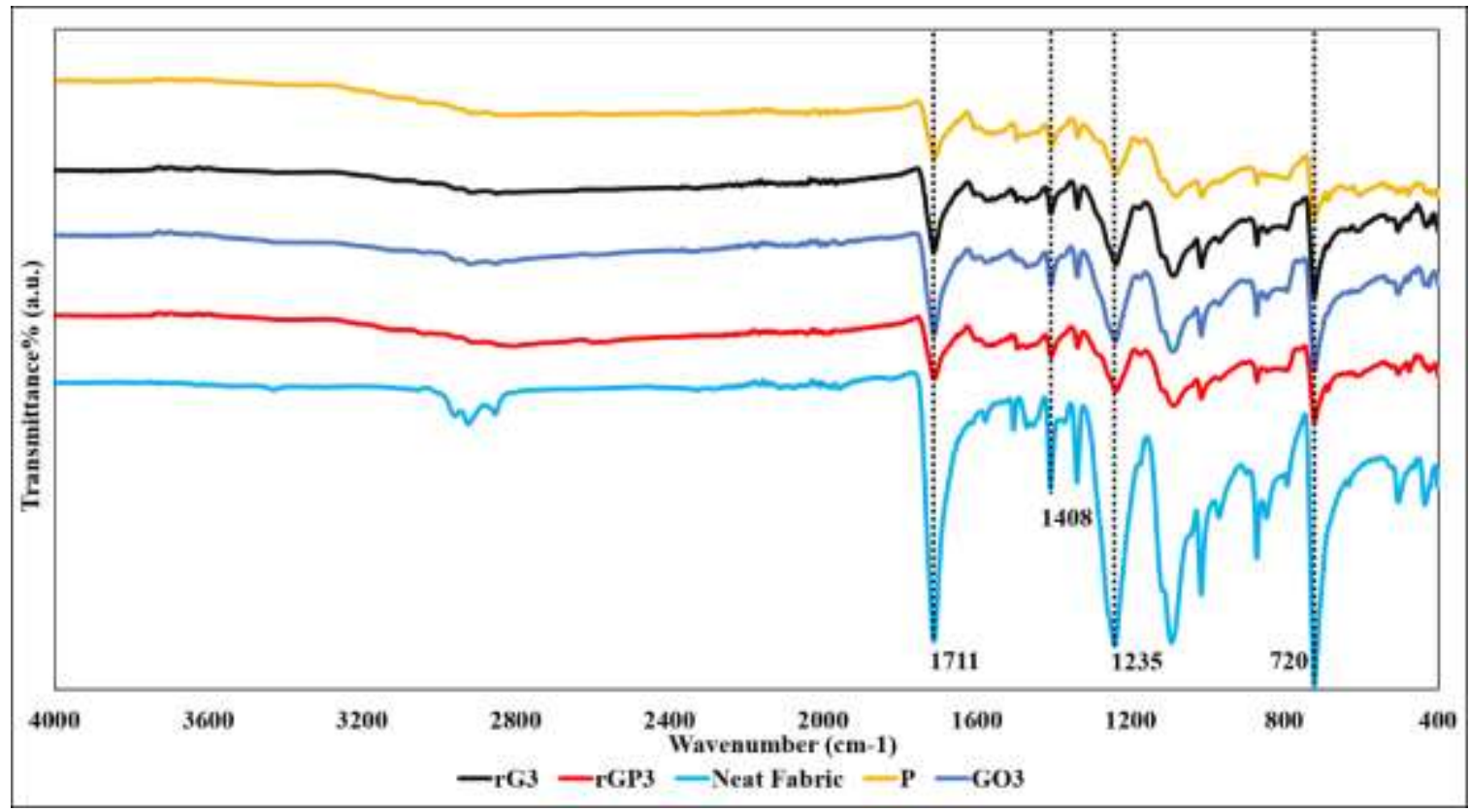

Figure 1. FT-IR spectra of neat and coated polyester fabrics

Light absorption characteristics of raw and coated fabrics were characterized with spectrophotometer between $350 \mathrm{~nm}-750 \mathrm{~nm}$ wavelength (Figure 2). UV-VIS absorbance spectra of neat polyester fabric showed regular absorbance curve with absorbance increase under $400 \mathrm{~nm}$ wavelength. However, graphene and/or PANI coated fabrics showed serrated and regular horizontal lines thorough the visible spectrum. Heterogeneity of graphene or PANI at nano or sub-micro scale caused this irregularity. The rG1 sample showed only different UV-VIS absorbance curve with lower light absorbance. Furthermore, PANI coated fabric showed a peak at $520 \mathrm{~nm}$. This peak is the characteristic peak of polyaniline, according to literature [30].

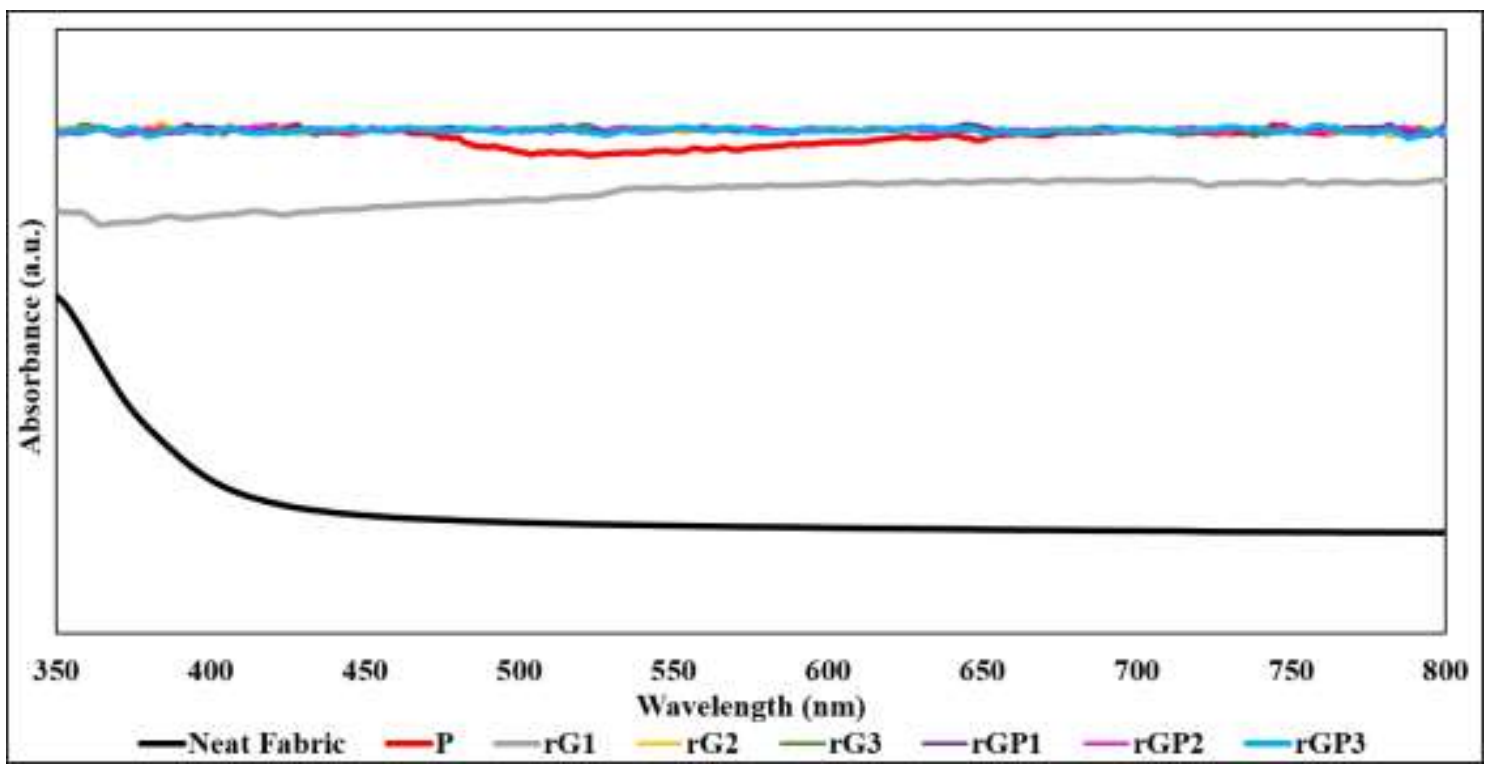

Figure 2. UV-VIS absorption curve of neat and coated fabrics 
DSC analysis were performed in order to investigate the $\mathrm{rGO}$ and/or PANI effect on thermal properties of fabrics. DSC experiments were performed with $3{ }^{\circ} \mathrm{C} / \mathrm{min}$ heating rate under nitrogen atmosphere. All samples gave a melting dip at $250^{\circ} \mathrm{C}$ independently from coating amount and material (Figure 3). Additionally, PANI and rGO/PANI coated fabrics showed two dips at $139{ }^{\circ} \mathrm{C}$ and $210{ }^{\circ} \mathrm{C}$ which are caused by glass transition and melting of PANI, respectively [31].

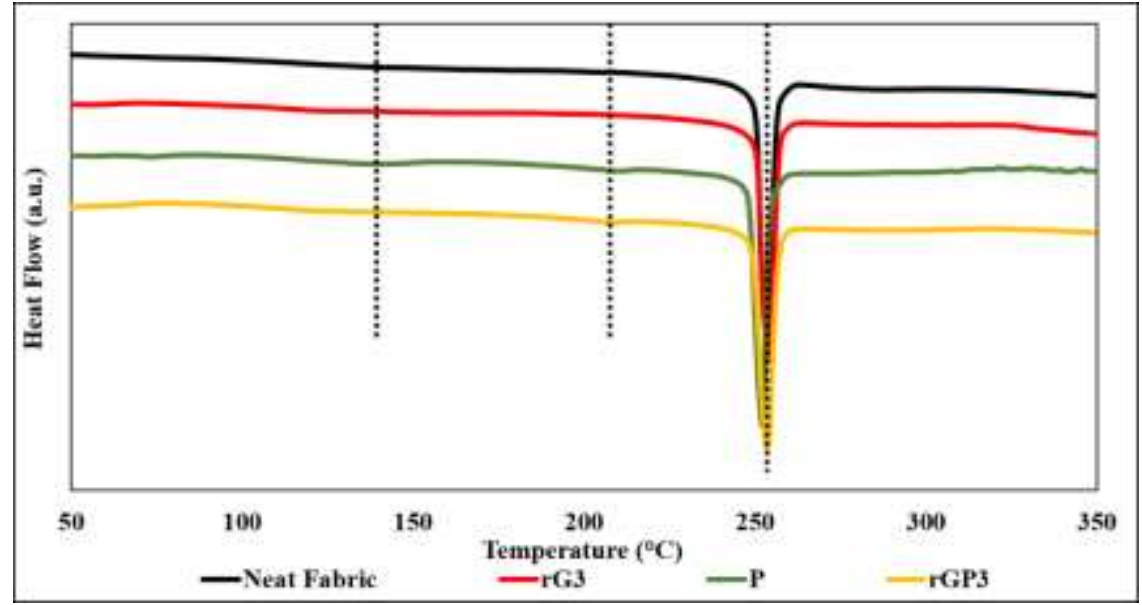

Figure 3. DSC curves of neat and coated fabrics

The woven fabrics were investigated by SEM technique to show fiber morphology, fabric surface and coating morphology. According to SEM images (Figure 3) of neat elastic polyester fabric, fabric surface was relatively rough, and diameter fibers were about $10 \mu \mathrm{m}$. Because of that, graphene sheets cannot completely wrap the fibers due to its a few micrometers of planar sizes. Graphene coating was consisting of partially wrapping of fibers, as shown in SEM images of rGO coated polyester fabric (Figure 4). Graphene sheets although could not wrap the woven fiber bundles, it could wrap the single fibers and wrapped fiber structure provided the electrical pathway formation. Moreover, graphene sheets could infuse the woven structure as an advantage of dip-coating method. SEM images also showed that the aniline successfully synthesized onto the fiber or rGO coated fiber surface (Figure 5).

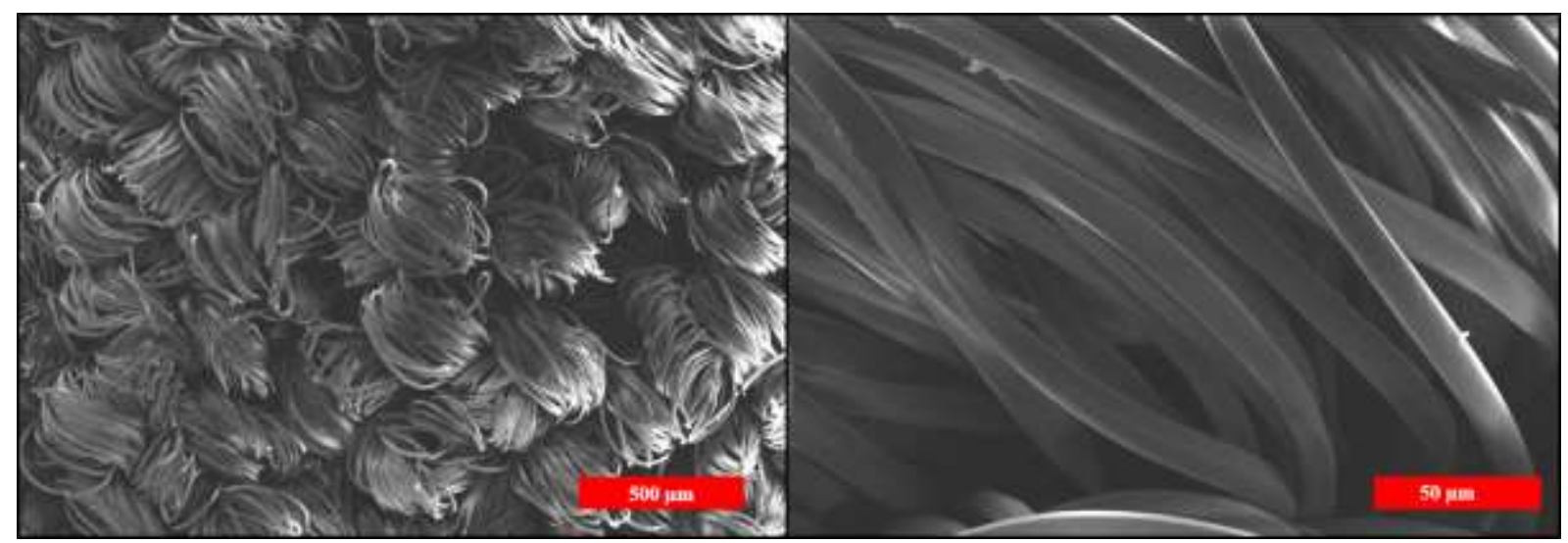

Figure 4. SEM images of neat polyester fabric 


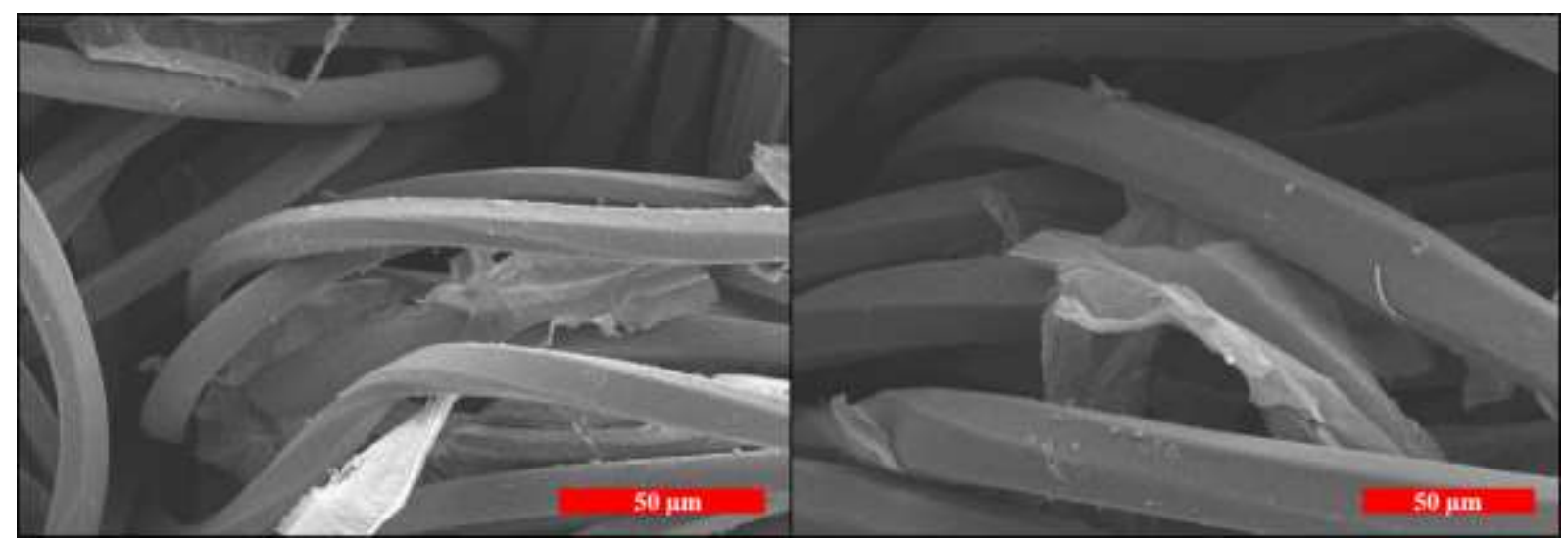

Figure 5. SEM images of rGO coated polyester fabrics

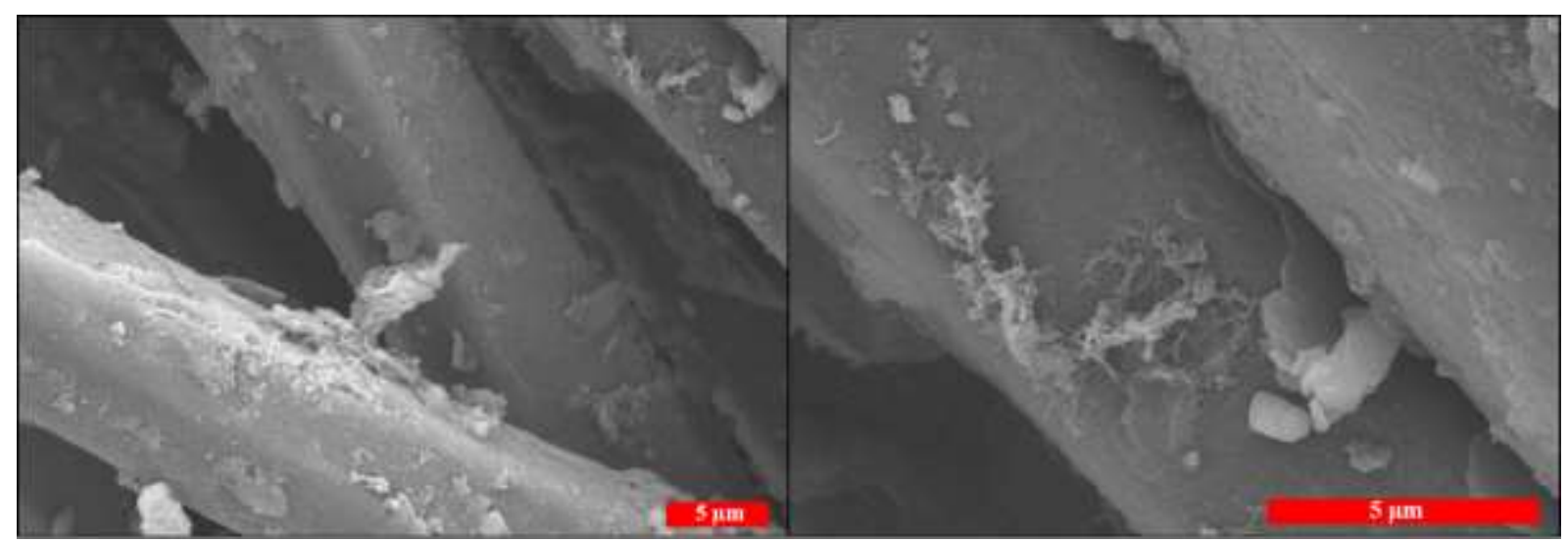

Figure 6. SEM images of PANI+rGO coated polyester fabrics

Neat fabric was an elastic and woven structure. Although elastic materials have regaining the original shape ability, because of high mechanical force and nature of polyester fibers, plastic deformation is possible for elastic polyester fabric. Because of that, a tensile test was performed on neat fabric in order to determine the elastic region. The fabric with $6 \mathrm{~cm} \times 15 \mathrm{~cm}$ sizes is used for tensile test and test speed was $5 \mathrm{~mm} / \mathrm{s}$. Fabric sample also boiled at $95{ }^{\circ} \mathrm{C}$ to achieve structural equality with coated samples since the coated fabrics were exposed to heat in GO reduction process. As the result of the tensile tests, it was determined that the uncoated fabrics were in the elastic region up to $100 \%$ elongation and were exposed to plastic deformation at elongations above $100 \%$ (Figure 7). Since the range in which the fabric sensor can be used will be until the end of the elastic region, electromechanical tests were continued until the fabric reached $100 \%$ elongation value. 


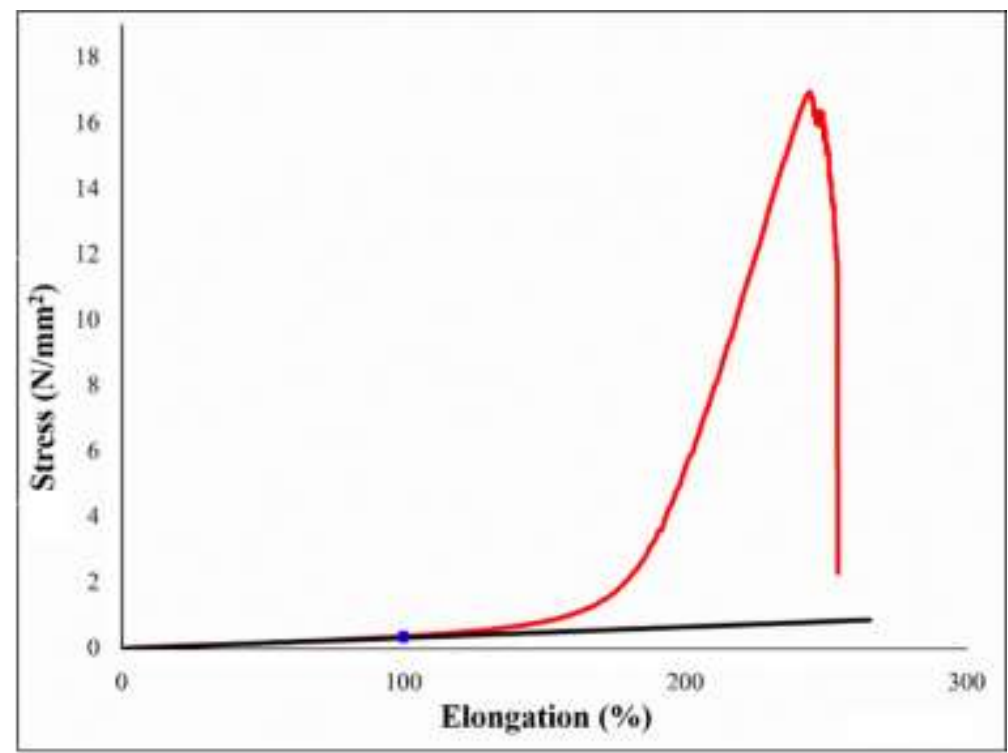

Figure 7. Stress-strain curve of neat fabric sample

In electromechanical tests, distance between shoulders were $10 \mathrm{~cm}$. Copper tapes were mounted at the two ends of sample as top and bottom electrodes and electrical contacts connected to KEITHLEY 2400 multimeter. As mentioned in characterization topic, sample was elongated 5\% and it stopped for 5 seconds. Resistivity value on multimeter's screen was manually recorded. This cycle was maintained until the total elongation reaches to $100 \%$.

Firstly, PANI coated sample were electromechanically tested. It was observed that the resistance increased abruptly and the resistance value could not be read after $40 \%$ elongation, which also means that the resistance increased above $1 \mathrm{G} \Omega$ (Figure 8). The resistance changes up to this level was determined as $2488 \%$. The maximum resistance value was measured as $68 \mathrm{M} \Omega$. It was predicted that the reason for this abrupt increase and the loss of conductivity of the fabric at $40 \%$ elongation was due to the hard and fragile behavior of PANI [32].

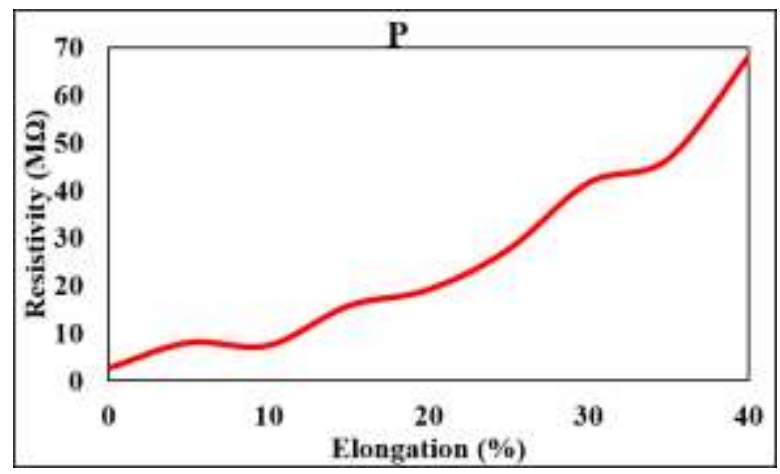

Figure 8. Elongation-electrical resistivity curve of PANI coated fabric

In rGO coated fabrics, it was determined that rG1 sample was quite insulating structure that it did not show any resistance value. It was observed that fabrics with initial resistances directly proportional to the amount of coating were produced in 2-layer and 3-layer rGO coated fabrics. It was determined that the electrical resistance of $\mathrm{rG} 2$ and $\mathrm{rG} 3$ fabrics increased by $12.5 \%$ and $30.4 \%$, respectively, at the end of $100 \%$ elongation (Figure 9). Maximum electrical resistance of the rGO coated fabrics was measured 
to be approximately $32 \mathrm{M} \Omega$. In the rGO-coated samples, a slower increase in resistance was observed with elongation movement, unlike the PANI-coated sample. The fact that the rGO particles were up to a few microns in width and length ensured that the fibers were tightly wrapped, the conductivity was higher than PANI, and the interface between fiber and conductive material was more stable [33].

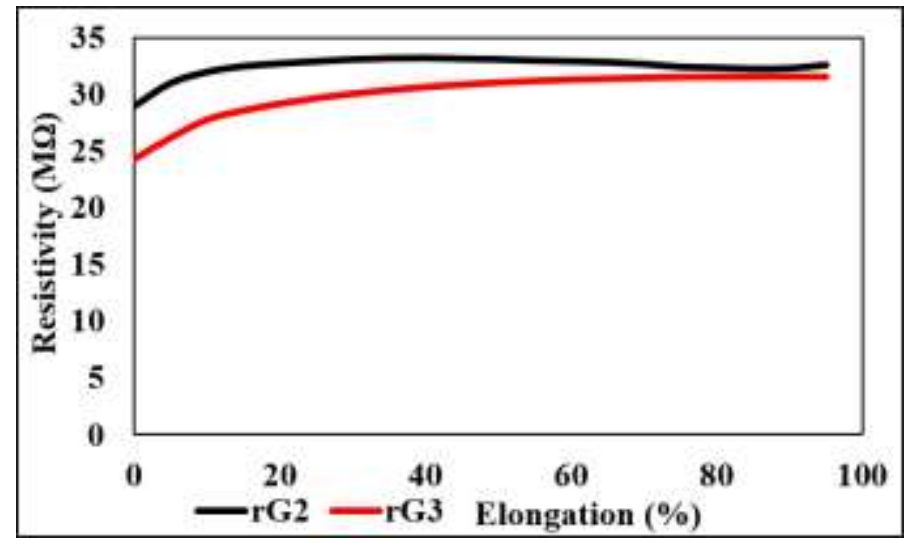

Figure 9. Elongation-electrical resistivity curve of rGO coated fabric

In electromechanical tests for 1 layer, 2 layer, and 3 layer rGO and fabric sensors coated with PANI on top of it, a more conductive structure was obtained compared to those coated with PANI or rGO. Under mechanical deformation, rG2 and rG3 samples and rGP2 and rGP3 samples showed similar curves, while rGP1 sample showed a non-linear curve. For rGP1, rGP2 and rGP3, the changes in resistance at the end of $100 \%$ elongation were measured as $191 \%, 87 \%$ and $97 \%$, respectively (Figure 10). The synergistic effect of PANI and graphene derivatives in PANI/rGO samples was confirmed [34].

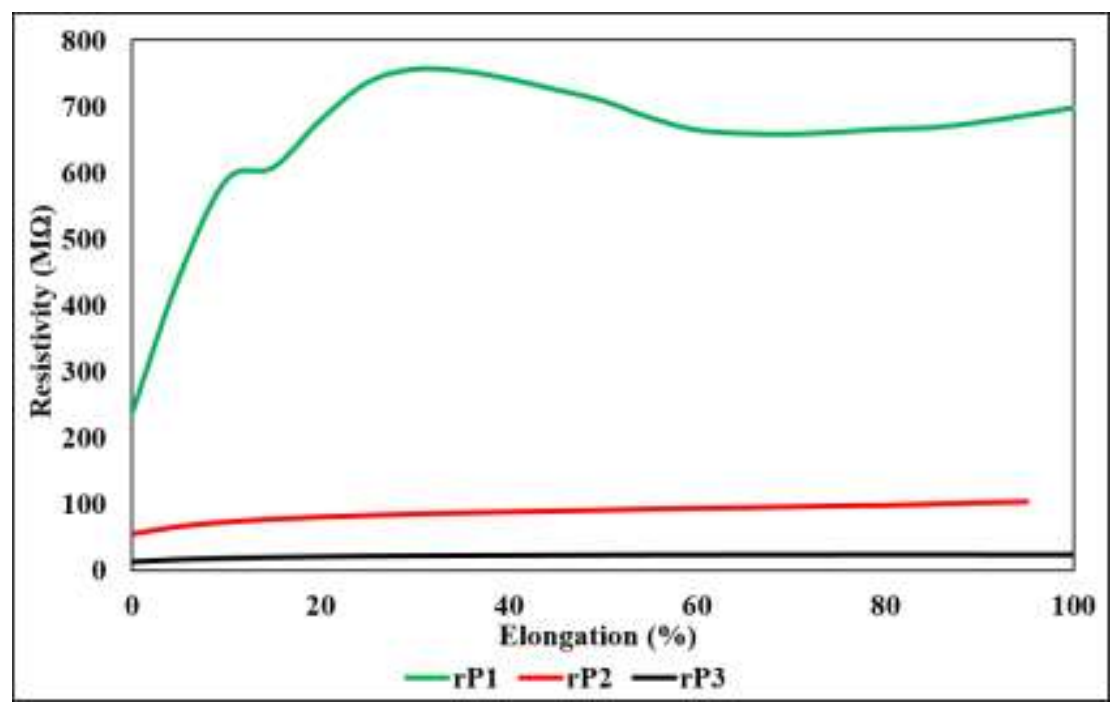

Figure 10. Elongation-electrical resistivity curve of rGO coated fabric

\section{CONCLUSIONS}

In electro-mechanical tests on conductive coated elastic fabrics, it was observed that the type and configuration of the coating material gave different responses in the detection of transformation. While a striking change in resistance to unit strain was observed in the PANI coated fabric, it was observed 
that the intensity of this reaction decreased with the inclusion of rGO in the structure. On the other hand, it was determined that a signal can be received from the PANI coated sensor fabric up to a limited amount of elongation, and it can be used in a wider strain area in $\mathrm{rGO}$ and PANI/rGO coated structures despite the lower signal strength. The reason for this situation is the flexibility and particle sizes of graphene and its derivatives, and the hard and brittle structure of PANI. In the light of this study the reduction of fabric sensors with different reduction methods of GO in the following studies, coating with different methods and the application of different conductive polymer coatings on rGO to wearable electronics by comparing them among themselves are planned in future studies.

\section{CONFLICT OF INTEREST}

The authors stated that there are no conflicts of interest regarding the publication of this article.

\section{REFERENCES}

[1] $\mathrm{Hu}$ J, Meng H, Li G and Ibekwe SI. A review of stimuli-responsive polymers for smart textile applications. Smart Materials and Structures, 2012; 21(5): 053001.

[2] Jin LN, Shao F, Jin C, Zhang JN, Liu P, Guo MX and Bian SW. High-performance textile supercapacitor electrode materials enhanced with three-dimensional carbon nanotubes/graphene conductive network and in situ polymerized polyaniline. Electrochimica Acta, 2017; 249: 387394.

[3] Altin Y and Çelik Bedeloğlu A. Energy storage textile. Advances in Functional and Protective Textiles, 2020; 493-529.

[4] Ünsal ÖF, Sezer Hiçyilmaz A, Yüksel Yilmaz AN, Altin Y, Borazan İ and Çelik Bedeloğlu A. 2020; Energy-generating textiles.

[5] Yu P, Li Y, Zhao X, Wu L and Zhang Q. Graphene-wrapped polyaniline nanowire arrays on nitrogen-doped carbon fabric as novel flexible hybrid electrode materials for high-performance supercapacitor. Langmuir, 2014; 30(18): 5306-5313.

[6] Yue B, Wang C, Ding X and Wallace GG. Polypyrrole coated nylon lycra fabric as stretchable electrode for supercapacitor applications. Electrochimica Acta, 2012;68: 18-24.

[7] Yang Z, Pang Y, Han XL, Yang Y, Yang Y, Ling J, Jian M, Zhang Y and Ren TL. Graphene Textile Strain Sensor with Negative Resistance Variation for Human Motion Detection. ACS Nano, 2018; 12(9): 9134-9141.

[8] Yang T, Jiang X, Zhong Y, Zhao X, Lin S, Li J, Li X, Xu J, Li Z and Zhu H. A wearable and highly sensitive graphene strain sensor for precise home-based pulse wave monitoring. ACS Sensors, 2017;2(7): 967-974.

[9] Hill EW, Vijayaragahvan A and Novoselov K. Graphene sensors. IEEE Sensors Journal, 2011; 11(12): $3161-3170$.

[10] Bedeloğlu A and Taş M. Graphene and Its Production Methods. Afyon Kocatepe University Journal of Sciences and Engineering, 2016;16(3): 544-554.

[11] Sadak O, Wang W, Guan J, Sundramoorthy A K, and Gunasekaran S. $\mathrm{MnO}_{2}$ Nanoflowers 
Deposited on Graphene Paper as Electrode Materials for Supercapacitors. ACS Applied Nano Materials, (2019);2(12): 4386-4394

[12] Sadak O, Sundramoorthy A K, and Gunasekaran S. Facile and green synthesis of highly conductive graphene paper. Carbon, (2018);138: 108-117

[13] Ünsal OF, Altin Y and BEDELOĞLU A. Dielectrıc properties of polyaniline-functionalized carbon nanotube/pdms anocomposites. Uludağ University Journal of the Faculty of Engineering, 2020; 25(2): 861-874.

[14] Altin Y, Unsal OF. and Celik Bedeloglu A. Fabrication and characterization of polyaniline functionalized graphene nanosheets (GNSs)/polydimethylsiloxane (PDMS) nanocomposite films, 2021; Https://Doi.Org/10.1177/09673911211023941.

[15] Sadak O, Prathap M U A, and Gunasekaran S. Facile fabrication of highly ordered polyanilineexfoliated graphite composite for enhanced charge storage. Carbon, (2019);144: 756-763

[16] Molina J, Esteves MF, Fernández J, Bonastre J and Cases F. Polyaniline coated conducting fabrics. Chemical and electrochemical characterization. European Polymer Journal, 2011; 47(10): 2003-2015.

[17] Molina J, del Río, AI, Bonastre J and Cases F. Electrochemical polymerisation of aniline on conducting textiles of polyester covered with polypyrrole/AQSA. European Polymer Journal, 2009; 45(4): 1302-1315.

[18] Saeb MR and Zarrintaj P. Polyaniline/graphene-based nanocomposites, In Fundamentals and Emerging Applications of Polyaniline, Elsevier, 2019; pp: 165-175.

[19] Akter Shathi M, Minzhi C, Khoso NA, Deb H, Ahmed A and Sai Sai W. All organic graphene oxide and Poly (3, 4-ethylene dioxythiophene) - Poly (styrene sulfonate) coated knitted textile fabrics for wearable electrocardiography (ECG) monitoring. Synthetic Metals, 2020; 263: 116329.

[20] Ünsal ÖF. Altın Y and Çelik Bedeloğlu A. Poly(vinylidene fluoride) nanofiber- based piezoelectric nanogenerators using reduced graphene oxide/polyaniline. Journal of Applied Polymer Science, 2020; 137(13): 48517.

[21] Shao F, Bian SW, Zhu Q, Guo MX, Liu S and Peng YH. Fabrication of Polyaniline/Graphene/Polyester Textile Electrode Materials for Flexible Supercapacitors with High Capacitance and Cycling Stability. Chemistry - An Asian Journal, 2016; 11(13): 19061912.

[22] Saberi Motlagh M and Mottaghitalab V. The charge transport characterization of the polyaniline coated carbon fabric as a novel textile based counter electrode for flexible dye-sensitized solar cell. Electrochimica Acta, 2017; 249: 308-317.

[23] Song P, He X, Xie M, Tao J, Shen X., Ji Z, Yan Z, Zhai L and Yuan A. Polyaniline wrapped graphene functionalized textile with ultrahigh areal capacitance and energy density for highperformance all-solid-state supercapacitors for wearable electronics. Composites Science and Technology, 2020;198: 108305. 
[24] Marcano DC, Kosynkin DV, Berlin JM, Sinitskii A, Sun Z, Slesarev A, Alemany LB, Lu W and Tour JM. Improved synthesis of graphene oxide. ACS Nano, 2010; 4(8): 4806-4814.

[25] Stankovich S, Dikin DA, Piner RD, Kohlhaas KA, Kleinhammes A, Jia Y, Wu Y, Nguyen SBT and Ruoff RS. Synthesis of graphene-based nanosheets via chemical reduction of exfoliated graphite oxide. Carbon, 2007; 45(7): 1558-1565.

[26] Li M, Huang X, Wu C, Xu H, Jiang P and Tanaka T. Fabrication of two-dimensional hybrid sheets by decorating insulating PANI on reduced graphene oxide for polymer nanocomposites with low dielectric loss and high dielectric constant. Journal of Materials Chemistry, 2012; 22(44): $23477-$ 23484 .

[27] Zhang C, He S, Wang D, Xu F, Zhang F and Zhang G. Facile fabricate a bioinspired Janus membrane with heterogeneous wettability for unidirectional water transfer and controllable oilwater separation. Journal of Materials Science, 2018;53(20): 14398-14411.

[28] Bhattacharya SS and Chaudhari SB. Study on Structural, Mechanical and Functional Properties of Polyester Silica Nanocomposite Fabric. International Journal of Pure and Applied Sciences and Technology, 2014; 21(1): 43-52.

[29] Hoghoghifard S, Mokhtari H and Dehghani S. Improving the conductivity of polyaniline-coated polyester textile by optimizing the synthesis conditions. Journal of Industrial Textiles, 2016; 46(2): 611-623.

[30] Ünsal ÖF. İletken polimer ve grafen oksitle fonksiyonelleştirilmiş nanolif tabanlı piezoelektrik nanojeneratörler, 2018.

[31] Sampreeth T, Al-Maghrabi MA, Bahuleyan BK and Ramesan MT. Synthesis, characterization, thermal properties, conductivity and sensor application study of polyaniline/cerium-doped titanium dioxide nanocomposites. Journal of Materials Science, 2018;53(1): 591-603.

[32] Tian M, Wang Y, Qu L, Zhu S, Han G, Zhang X, Zhou Q, M. Du and S. Chi, Electromechanical deformation sensors based on polyurethane/polyaniline electrospinning nanofibrous mats. Synthetic Metals, 2016; 219: 11-19.

[33] Ali MA, Umer R, Khan KA, Samad YA, Liao K and Cantwell W. Graphene coated piezo-resistive fabrics for liquid composite molding process monitoring. Composites Science and Technology, 2017;148: 106-114.

[34] Cheng X, Kumar V, Yokozeki T, Goto T, Takahashi T, Koyanagi J, Wu L and Wang R. Highly conductive graphene oxide/polyaniline hybrid polymer nanocomposites with simultaneously improved mechanical properties. Composites Part A: Applied Science and Manufacturing, 2016; 82: $100-107$. 